\title{
Patient-Specific Biomechanical Modeling of Ventricular Enlargement in Hydrocephalus from Longitudinal Magnetic Resonance Imaging
}

\author{
Yasheng Chen ${ }^{1,2}$, Zheng Fan $^{3}$, Songbai $\mathrm{Ji}^{4}$, \\ Joseph Muenzer ${ }^{5,6}$, Hongyu An ${ }^{1,2}$, and Weili Lin ${ }^{1,2}$ \\ ${ }^{1}$ Biomedical Research Imaging Center, Dept. of \\ ${ }^{2}$ Radiology, ${ }^{3}$ Neurology, ${ }^{5}$ Pediatrics and ${ }^{6}$ Genetics, \\ University of North Carolina at Chapel Hill, NC, 27599 \\ ${ }^{4}$ Thayer School of Engineering, Dartmouth College, Hanover, NH 03755 \\ \{yasheng_chen, zheng_fan, joseph_muenzer, \\ hongyuan, weili_lin\} @med.unc.edu, \\ songbai.ji@dartmouth.edu
}

\begin{abstract}
Ogden type of hyperelastic constitutive law has recently emerged in modeling ventricular enlargement in hydrocephalic brain with finite element method, but this material property for brain tissue has not been investigated in a patient-specific setting in hydrocephalus. Consequently, the accuracy of the simulated ventricular enlargement using this hyperelastic tissue property remains unknown. In this study, we evaluated this brain material model in four patients with communicating hydrocephalus under a small trans-mantle pressure difference (TPMD) between brain ventricle and subarachnoid space $(<1 \mathrm{mmHg})$. Based upon changes in ventricular geometries obtained with sequential MRI, we found that this hyper-elastic model has a great flexibility and accuracy in modeling ventricular enlargement (with errors less than $1 \mathrm{~mm}$ ). Our study supports the utility of this hyperelastic constitutive law for future hydrocephalus modeling and suggests that the observed ventricular enlargement in these patients may be caused by a slight increase in TMPD.
\end{abstract}

Keywords: Brain finite element modeling, Brain mechanics, Hydrocephalus, Hyper-elastic brain modeling, Finite element analysis, Ventricular enlargement.

\section{Introduction}

Hydrocephalus is defined as an active enlargement of brain ventricles caused by the impairment of cerebrospinal fluid (CSF) homeostasis. CSF is mainly produced by the choroid plexus in the lateral and third ventricles. After flowing into the fourth ventricle through aqueduct of Sylvius, CSF is reabsorbed in the subarachnoid space (SAS) through arachnoid granulations in the sagittal sinus. Hydrocephalus is referred to as communicating hydrocephalus when no apparent obstruction is presented within the brain ventricular system [1].

Finite element method (FEM) based simulation of brain ventricle enlargement started with a biphasic model for brain tissue. Nagashima et al employed Biot's consolidation 
theory to model brain tissue as a biphasic poroelastic medium in $2 \mathrm{D}$, and demonstrated that increased hydraulic conductivity may lead to periventricular CSF edema. The transmantle pressure difference between ventricle and SAS (TMPD) applied to the ventricular wall was $20 \mathrm{mmHg}$ [2]. Pena et al demonstrated that distribution of periventricular edema in acute hydrocephalus is highly related to not only the increased intra-ventricular pressure, but also the natural geometry of the ventricle [3]. Clatz et al combined the biphasic brain tissue model with a scalar model of CSF productionresorption cycle and used Monroe-Kellie assumption (the total volume of brain, CSF and blood is a constant due to the confinement of skull) to simulate the interaction between brain tissue and CSF production under the assumption of infinitesimal deformation [4]. As pointed out in [5], these works have limitations by using both linear elastic tissue property and linear geometrical analysis. More recently, Momjian and Bichsel introduced nonlinearity to this poroelastic model in 2D by varying the Young's modulus as a function of local parenchymal void ratio and relaxing the internal stress [6].

Different from the previously discussed biphasic tissue model, through testing porcine brain tissue, Miller et al have found that brain tissue deforms like a hyperelastic material with a linear viscoelasticity $[7,8]$. Due to the large time scale considered for ventricular enlargement, this model was simplified to an Ogden type of hyperelastic constitutive law and was first applied to simulate ventricular enlargement in hydrocephalus by Taylor and Miller [9]. Based upon this hyperelastic model, they suggested that the modulus in previous biphasic modeling of hydrocephalus may be too high, and a considerably lower modulus value of approximately 584Pa should be used [9]. In addition, based upon generic brain geometry, Dutta-Roy et al demonstrated that the Biot's consolidation theory based biphasic brain tissue model is not advantageous when compared to the hyperelastic model in simulating ventricular enlargement [5]. In this work, this hyperelastic model will be further evaluated in terms of deformation capability and accuracy in modeling ventricular enlargement in a patient-specific setting.

As pointed out in [10], one important limitation in current FEM-based numerical analyses in hydrocephalus is the lack of quantitative comparison between the FEM simulation and the actual patient-specific anatomical changes caused by hydrocephalus, which provides a critical feedback for us to gain more insights into brain mechanics in hydrocephalus. To address this limitation, we performed patientspecific geometrical modeling of ventricle enlargement using FEM simulation and the accuracy of the simulated deformation was assessed by using the same patient's sequential MRI as the ground truth. In particular, we sought to determine whether FEM simulation with this hyperelastic model using the parameters as in [5] can model the ventricular enlargement accurately especially under a small pressure loading. To the best of our knowledge, our study is the first 3D nonlinear FEM analysis of ventricular enlargement in hydrocephalus in a patient-specific setting.

\section{Methods}

\subsection{Image Acquisition and Preprocessing}

This is an institution review board (IRB) approved study and written consents were obtained prior to image acquisition. We included four patients with communicating 
hydrocephalus caused by lysosomal storage disorder. In these patients, ventricular enlargement is a chronic process developed in years. The T1 weighted images of these four patients were acquired 24 months, 28months, 17 months, and 57months apart, respectively. No treatment was administrated for these patients. The T1 weighted images from the later scans were registered onto the same patient's initial scan using the linear registration in FSL toolkit [11]. Ventricular segmentation was performed with an atlas based approach and manual editing was performed when missegmentation occurred with the altered T1 intensity due to the disease. The brain supporting structures including falx cerebri and tentorium cerebelli were manually delineated due to their low image contrast in T1. Tetrahedral meshes were generated using the segmented brain images with a constrained Delaunay process [12].

\subsection{Biomechanical Modeling}

The hyperelastic material model for the brain was originally proposed by Miller et al as a linear viscoelastic material to account for the nonlinear and strain rate dependent behavior of brain tissue during deformation [7,8]. Due to the large time scale considered for ventricular enlargement, this model was simplified to an Ogden type of hyerpelastic model assuming a potential quasi-static loading with zero strain rate [5]. The hyperelastic constitutive law for brain parenchyma is given as:

$$
W=2 \mu / \alpha^{2}\left(\lambda_{1}^{\alpha}+\lambda_{2}^{\alpha}+\lambda_{3}^{\alpha}\right)+\frac{1}{D}\left(J^{e l}-1\right)^{2},
$$

where $W$ is the potential function; $\lambda_{\mathrm{i}} \mathrm{s}$ are the principle stretches, $\mu$ is the initial shear modulus $(155.77 \mathrm{~Pa})$ and $\alpha$ is a material constant $(-4.7)[5,9] . \quad \mathrm{J}^{\mathrm{el}}$ is the elastic volume ratio and $D$ is a material coefficient inversely related to the initial bulk modulus. Effectively, this model has only three parameters including $\mu$, a, and $D$, and $\lambda_{\mathrm{i}} \mathrm{s}$ and $\mathrm{J}^{\mathrm{el}}$ are computed from nodal displacements. Poisson's ratio (PR) is computed as (6/ $(D \mu)-2) /(12 /(D \mu)+2)$. Previously, Dutta-Roy et al have chosen PR as 0.35 , 0.49 and 0.5 representing brain tissue as compressible, nearly incompressible, and fully incompressible materials [5]. It should be noted that even though we use a very fine mesh, potential locking may exist when the PR approximates 0.5 . We varied PR in a range of $[0.35,0.49]$ at a step size of 0.02 for all the four patients. The rigid supporting structures including falx cerebri and tentorium cerebelli were modeled as isotropic elastic material with a Young's modulus of $3.15^{*} 10^{7} \mathrm{~Pa}$ and a PR of 0.45 [13]. It is also worth pointing out that our study is the first to consider these supporting structures in hydrocephalus modeling even though the Young's modulus of these supporting structures is approximately 105 times higher than the initial Young's modulus of the hyperelastic brain tissue model $(\sim 584 \mathrm{~Pa})$.

We have applied both large $(\sim 10 \mathrm{mmHg})$ and small pressure loadings $(<1 \mathrm{mmHg})$ to the ventricular wall in FEM simulation. The large pressure loading is used to evaluate the capability of this hyperelastic constitutive law in modeling large deformation in patient-specific geometrical setting.

Even though all current FEM-based studies in hydrocephalus load the model with an assumed TMPD [2-6, 9], intriguingly, several experimental studies (including both patient and animal studies) did not detect such a pressure difference [14-16]. 
These results suggest that the assumed TMPDs $(\sim 20 \mathrm{mmHg})$ in previous FEM studies $[2,3]$ were too high and their FEM models may not truly represent in-vivo biological conditions. In our study, we assume that the maximal TMPD applied to the ventricular wall is with $1 \mathrm{mmHg}(133.47 \mathrm{~Pa})$ as that in [5], and such a small TMPD is expected to be within the experimental variations in [14-16]. We also varied TMPD in the range of $0.1-1 \mathrm{mmHg}$ (with a step size of $0.1 \mathrm{mmHg}$ ) jointly with different PRs discussed previously.

Two types of brain-skull boundary conditions have been used in previous hydrocephalus modeling studies. The majority employed the Dirichlet boundary condition by fixing brain mesh surface nodes in all degrees-of-freedom [2-4, 6, 9]. The only exception is a nonlinear boundary condition constraining the brain surface nodes on the bottom while allowing the remainder to move within the cranial cavity as an arbitrary $3 \mathrm{~mm}$ gap between brain and skull [17]. This boundary condition was originally designed to account for brain shift due to the loss of CSF upon craniotomy for brain tumor resection. Since all subjects in this study had an intact skull, we employed the Dirichlet boundary condition accordingly.

\subsection{Evaluation of Simulation Accuracy}

The distance from one node in the simulated ventricular surface to the ground truth geometry (the ventricular surface from the later scan) was defined as the minimal distance from this node to all the triangular patches consisting of the ground truth ventricle (Eq. [2]).

$$
D(i)=\min \operatorname{dist}\left(\vec{x}_{l}, \operatorname{Tr}_{j}\right) i \in M, j \in N
$$

where $D(i)$ is the shortest distance from node $i$ on the simulated ventricular surface to all the triangular patches (Tri) on the ground truth ventricle from the later MRI. $M$ is the total number of nodes in the simulated ventricular surface and $N$ represents the total number of triangular patches on the ground truth ventricle. We further compute the weighted sum of $D(i)$ from all the nodes as a measure of error of the simulation (Eq. (3)). The weight for one node was computed as the ratio of 1/3 of the total area of all the neighboring triangular patches over the total area of the entire simulated ventricular surface (Eq. (3)). The purpose is to account for the area differences of the triangular patches after mesh is deformed.

$$
w_{i}=\sum_{j \in N_{i}} 1 / 3 * \text { Area }_{j} / \sum_{j=1}^{N} \text { Area }_{j} ; D_{e}=\sum_{i=1}^{M} w_{i} D(i)
$$

$N_{i}$ is the number of neighboring patches to node $i$, and $A r e a_{j}$ is the area of the triangular patch $j$ on the surface. For reference, the same measure was also computed between the initial and the ground truth ventricles of the same patient (referred to as $D_{\text {init }}$ ).

\section{Results}

\subsection{Deformation Capability of Hyperelastic Modeling}

The registered images from the later scans to the initial scans are given in Fig. 1 for all four patients. The rendered ventricular surfaces from patient 1 are given for the initial 
(Fig. 2(a)) and later (Fig. 2(b), the ground truth) scans. The ventricular enlargement was especially apparent as the thickening of the frontal and occipital horns of the lateral ventricle (indicated by the arrows in Fig. 2(a) and 2(b)). Simulated ventricular surfaces with two sets of TMPD and PR are given in Fig. 2(c) and Fig. 2(d), respectively.

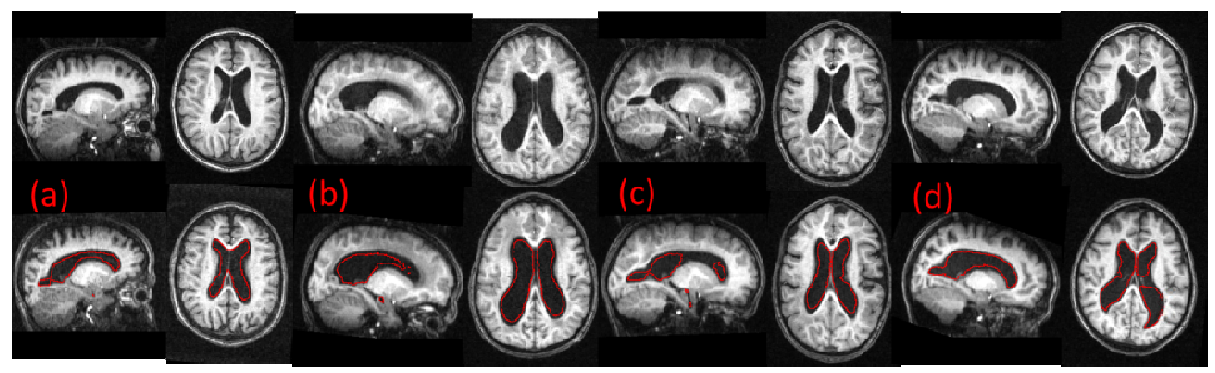

Fig. 1. T1 images from the four patients with the ventricle contour (red curves) from the initial visit (top panel) superimposed on the T1 images from the later visit (bottom panel)

In order to demonstrate the capability of this hyperelasticity constitutive law in modeling an extremely large deformation, we applied a TMPD of $10 \mathrm{mmHg}$ with a PR of 0.35 to determine whether the FEM analysis using this hyperelastic model is able to converge, because a TMPD of $4.0 \pm 3.6 \mathrm{mmHg}$ was reported in patients with hydrocephalus before [18]. The simulations converged at the TMPDs of $9.57 \mathrm{mmHg}$ (Fig 2 (d) for patient1), $10 \mathrm{mmHg}, 9.87 \mathrm{mmHg}$ and $10 \mathrm{mmHg}$ for these four patients, respectively. Thus, even without assorting to an adaptive numerical technique such as arbitrary Lagrangian and Eulerian FEM [19], this hyperelastic model for brain tissue allows us to simulate extremely large ventricular enlargement.

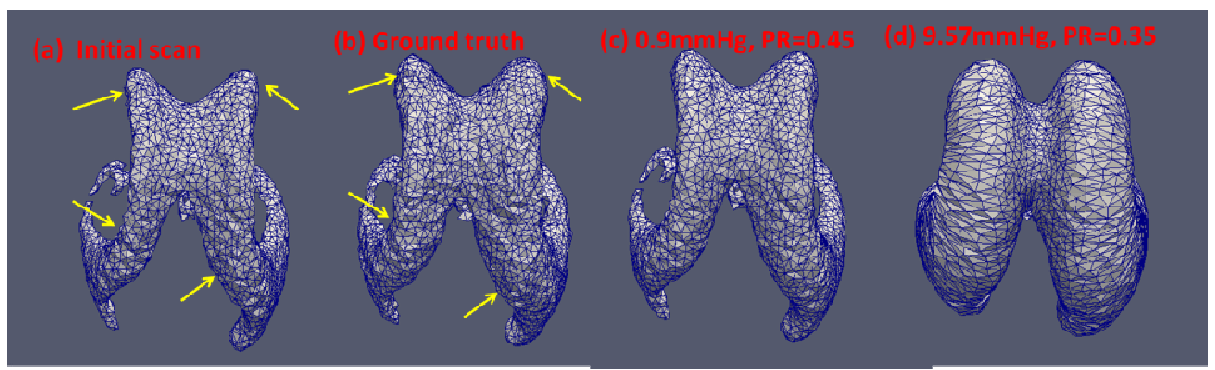

Fig. 2. The rendered ventricle shapes at the initial (a) and later (b) scans and from simulations with various conditions (c and d)

\subsection{Accuracy of FEM Simulation}

We varied PR and TMPD to minimize the fitting errors. In the 80 simulations for each patient performed with ABAQUS, the errors (computed via Eq. (3)), and the combinations of TMPD and PR having the least error for all patients are given in Fig. 3. The minimal errors were found at $(\mathrm{TMPD}=0.9 \mathrm{mmHg}, \mathrm{PR}=0.45),(\mathrm{TMPD}=1 \mathrm{mmHg}$, 
$\mathrm{PR}=0.45),(\mathrm{TMPD}=1 \mathrm{mmHg}, \mathrm{PR}=0.45)$ and $(\mathrm{TMPD}=0.6 \mathrm{mmHg}, \mathrm{PR}=0.41)$ for these four patients, respectively. The minimal errors $D_{e}$ for the simulated ventricular surfaces were $(0.773 \mathrm{~mm}, 0.983 \mathrm{~mm}, 0.940 \mathrm{~mm}, 0.718 \mathrm{~mm})$, which were $(49.7 \%, 48.4 \%$, $51.1 \%, 48.8 \%)$ of $D_{\text {init }}(1.554 \mathrm{~mm}, 2.031 \mathrm{~mm}, 1.841 \mathrm{~mm}, 1.472 \mathrm{~mm})$. For instance, the best approximation for patient 1 (Fig. 2(c)) bears a very high resemblance to the ground truth (Fig. 2(b)). The distance maps to the ground truth geometry from the initial scan (Fig. 4(a) and (c)) and the best simulation (Fig. 4(b) and (d)) in both dorsal and ventral views are rendered in Fig. 4. In Fig. 5, the $2 \mathrm{D}$ contours from the best simulations and their corresponding ground truth geometries were overlaid on the later MRI in three orthogonal slice locations for these four patients.
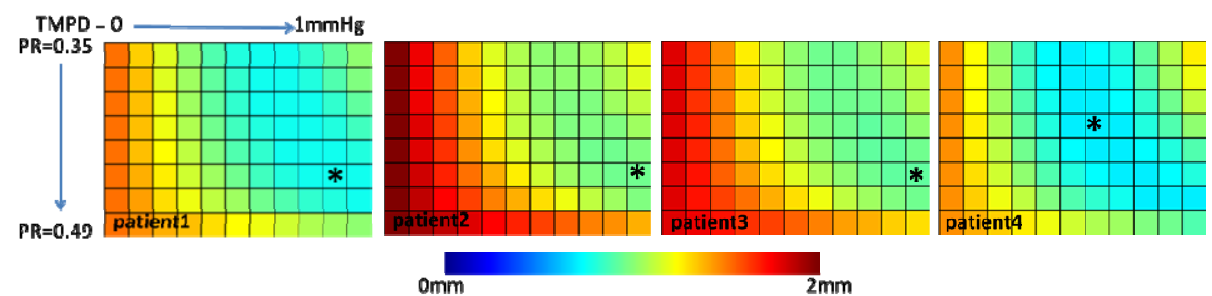

Fig. 3. The errors from the 80 simulations for the four patients with different combinations of TMPD and PR (with * indicating the parametric pair with the least error)

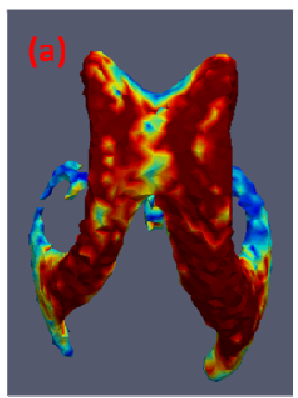

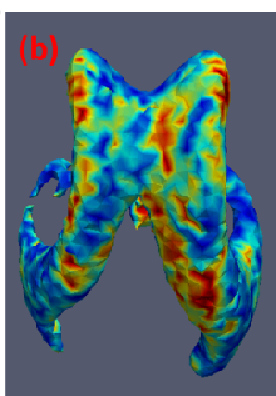

Omm

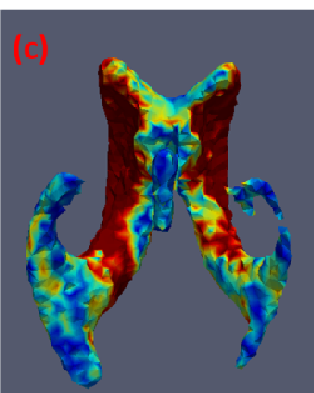

$2 \mathrm{~mm}$

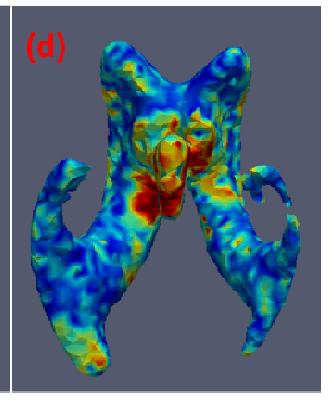

Fig. 4. The rendered maps of $D_{\text {init }}$ (a and c) and $D_{e}$ (b and d) for patient 1

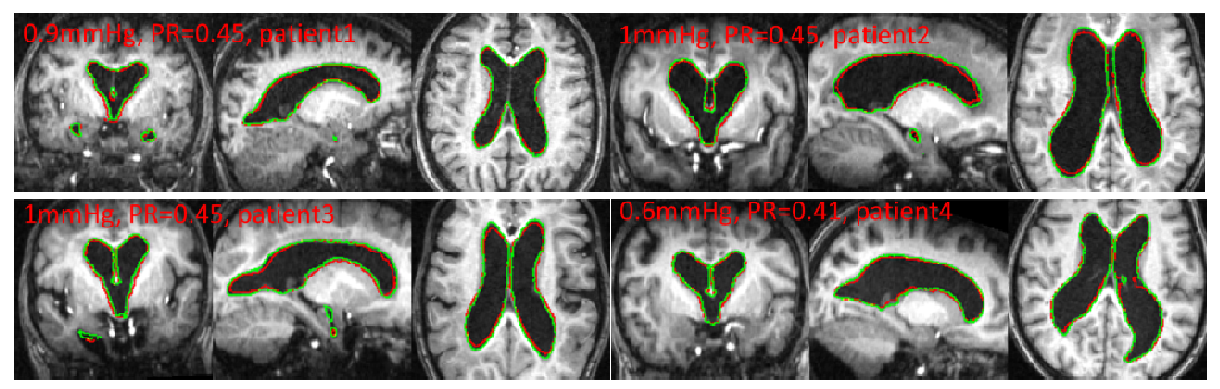

Fig. 5. The 2D contours comparing the ventricular boundaries obtained from the best simulation result (red) and the ground truth (green) super-imposed on the later MR scans 


\section{Conclusions}

Our initial experience with the hyperelastic modeling supports its future application in investigating hydrocephalus. We have demonstrated that with the previously suggested parameters, this model is not only able to approximate the deformation observed in these four patients with high accuracy (less than $1 \mathrm{~mm}$ in error), but also flexbile enough to achieve large deformations even under a large TMPD in line with previous experimental reports $(4.0 \pm 3.6 \mathrm{mmHg}[18])$.

Our results suggest that the investigation of the mechanical factors in communicating hydrocephalus may warrant the investigation of the existence of a small TMPD, which may be able to explain the controversies among some of the previous hydrocephalus studies. For instance, the existence of TMPD in hydrocephalic patients with ventricular enlargement has been reported in $[18,20]$ but not in [14-16]. We speculate that such a small TMPD as in communicating hydrocephalus may not be readily detected in these experiments due to instrumental variations.

Limitations in our study are noted. Measurements of TMPD and tissue property in these patients were not conducted due to the invasive nature of the involved procedures. Evidence from magnetic resonance elastography suggests that hydrocephalic brain tissue may be more compliant due to the weakened mechanical structures [21, 22]. If this is the case for our patients, even though we may have underestimated the ventricular enlargement under the same TMPD, the major conclusion that a small TMPD may leads to ventricular dilation in these patients remains to be true. Due to the same limitation, we did not consider the potential temporal changes and spatial inhomogeneity of brain tissue property. We varied Poisson's ratio to account for deviation from the originally proposed model parameters. At last, in the initial scans of these patients, brain tissue may have already been strained due to ventricular enlargement and our analysis may not start from an ideal tension-free setting.

\section{References}

1. Rekate, H.L.: A contemporary definition and classification of hydrocephalus. Semin. Pediatr. Neurol. 16, 9-15 (2009)

2. Nagashima, T., Tamaki, N., Matsumoto, S., Horwitz, B., Seguchi, Y.: Biomechanics of hydrocephalus: a new theoretical model. Neurosurgery 21, 898-904 (1987)

3. Pena, A., Bolton, M.D., Whitehouse, H., Pickard, J.D.: Effects of brain ventricular shape on periventricular biomechanics: a finite-element analysis. Neurosurgery 45, 107-116; discussion 116-108 (1999)

4. Clatz, O., Litrico, S., Delingette, H., Paquis, P., Ayache, N.: Dynamic model of communicating hydrocephalus for surgery simulation. IEEE Trans. Biomed. Eng. 54, 755-758 (2007)

5. Dutta-Roy, T., Wittek, A., Miller, K.: Biomechanical modeling of normal pressure hydrocephalus. J. Biomech. 41, 2263-2271 (2008)

6. Momjian, S., Bichsel, D.: Nonlinear poroplastic model of ventricular dilation in hydrocephalus. J. Neurosurg. 109, 100-107 (2008) 
7. Miller, K., Chinzei, K.: Constitutive modelling of brain tissue: experiment and theory. J. Biomech. 30, 1115-1121 (1997)

8. Miller, K.: Constitutive model of brain tissue suitable for finite element analysis of surgical procedures. J. Biomech. 32, 531-537 (1999)

9. Taylor, Z., Miller, K.: Reassessment of brain elasticity for analysis of biomechanisms of hydrocephalus. J. Biomech. 37, 1263-1269 (2004)

10. Rekate, H.L.: The usefulness of mathematical modeling in hydrocephalus research. Childs. Nerv. Syst. 10, 13-18 (1994)

11. Smith, S.M., Jenkinson, M., Woolrich, M.W., Beckmann, C.F., Behrens, T.E., JohansenBerg, H., Bannister, P.R., De Luca, M., Drobnjak, I., Flitney, D.E., Niazy, R.K., Saunders, J., Vickers, J., Zhang, Y., De Stefano, N., Brady, J.M., Matthews, P.M.: Advances in functional and structural MR image analysis and implementation as FSL. Neuroimage 23(suppl. 1). S208-S219 (2004)

12. Si, H.: Adaptive tetrahedral mesh generation by constrained Delaunay refinement. Int. J. Numer. Meth. Engrg. 75, 856-880 (2008)

13. Kleiven, S.: Predictors for traumatic brain injuries evaluated through accident reconstructions. Stapp. Car. Crash. J. 51, 81-114 (2007)

14. Penn, R.D., Lee, M.C., Linninger, A.A., Miesel, K., Lu, S.N., Stylos, L.: Pressure gradients in the brain in an experimental model of hydrocephalus. J. Neurosurg. 102, 10691075 (2005)

15. Shapiro, K., Kohn, I.J., Takei, F., Zee, C.: Progressive ventricular enlargement in cats in the absence of transmantle pressure gradients. J. Neurosurg. 67, 88-92 (1987)

16. Stephensen, H., Tisell, M., Wikkelso, C.: There is no transmantle pressure gradient in communicating or noncommunicating hydrocephalus. Neurosurgery 50, 763-771; discussion 771-763 (2002)

17. Wittek, A., Miller, K., Kikinis, R., Warfield, S.K.: Patient-specific model of brain deformation: application to medical image registration. J. Biomech. 40, 919-929 (2007)

18. Conner, E.S., Foley, L., Black, P.M.: Experimental normal-pressure hydrocephalus is accompanied by increased transmantle pressure. J. Neurosurg. 61, 322-327 (1984)

19. Chen, Y., Ji, S., Wu, X., An, H., Zhu, H., Shen, D., Lin, W.: Simulation of brain mass effect with an arbitrary Lagrangian and Eulerian FEM. Med. Image. Comput. Comput. Assist. Interv. 13, 274-281 (2010)

20. Hoff, J., Barber, R.: Transcerebral mantle pressure in normal pressure hydrocephalus. Arch. Neurol. 31, 101-105 (1974)

21. Streitberger, K.J., Wiener, E., Hoffmann, J., Freimann, F.B., Klatt, D., Braun, J., Lin, K., McLaughlin, J., Sprung, C., Klingebiel, R., Sack, I.: In vivo viscoelastic properties of the brain in normal pressure hydrocephalus. NMR Biomed. 24(4), 385-392 (2011)

22. Freimann, F.B., Streitberger, K.J., Klatt, D., Lin, K., McLaughlin, J., Braun, J., Sprung, C., Sack, I.: Alteration of brain viscoelasticity after shunt treatment in normal pressure hydrocephalus. Neuroradiology 54(3), 189-196 (2011, 2012) 